\title{
Um arcabouço que enfatiza a retroação a contextos de erro na solução de problemas
}

\author{
Diego Marczal \\ Programa de Pós-graduação em Informática \\ UFPR - Universidade Federal do Paraná \\ diego@inf.ufpr.br
}

\author{
Alexandre Direne \\ Departamento de Informática \\ UFPR - Universidade Federal do Paraná \\ alexd@inf.ufpr.br
}

\begin{abstract}
Resumo Inicialmente, são apresentados os aspectos que justificam a necessidade de se projetar $e$ implementar um controlador genérico de interatividade que pode ser aplicado e reutilizado por diversos objetos de aprendizagem. Os principais objetivos do controlador são detalhados em termos de acesso ao conteúdo e como ele enfatiza aspectos meta-cognitivos envolvidos nas tarefas típicas de aprendizagem. Além disso, apresenta-se uma nova abordagem para utilizar os erros cometidos pelo aprendiz como uma ferramenta eficiente para reparar e construir o seu conhecimento. Ao final, a arquitetura e a implementação do arcabouço denominado controlador de acesso reflexivo e retroativo indexado por erros (CARRIE) têm seus módulos principais descritos e exemplificados.
\end{abstract}

Palavras-Chave: Objetos de Aprendizagem, ambientes interativos de aprendizagem, retroação a contexto de erros.

\begin{abstract}
Initially, the paper presents the main aspects that justify the need for the design and implementation of a generic interactivity controller that can be applied and reused by a wide range of educational objects. The main objectives of the controller are detailed in terms of content access and the way it emphasizes meta-cognitive aspects involved with typical learning tasks. Moreover, it presents a new approach to use the errors made by the apprentice as a effective tool to repair and build your knowledge. At the end, the architecture and implementation of a framework called CARRIE (access Reflective and retroactive controller indexed by error) have their modules described and exemplified.
\end{abstract}

Keywords: Learning Objects, interactive learning environments, retroaction to the context of errors.

\section{Introdução}

Atualmente, vários objetos de aprendizagem (OA) e simuladores têm sido desenvolvidos para apoiar tarefas de visualização de conceitos e solução de problemas em diversas áreas do conhecimento humano (e.g., Matemática e Física). Por iniciativa coordenada do governo federal (MEC/SEED), boa quantidade desses 
objetos está disponível em bases de acesso público e gratuito. Esses $\mathrm{OA}$ possuem muitas características em comum, sendo que as principais estão relacionadas com a interface gráfica de acesso aos seus conteúdos educacionais. Todavia, a principal limitação deles está na ausência de um controlador genérico de interatividade que se aplique a todos os OA de maneira reutilizável e reconfigurável pelo autor do material de simulação e de solução de problemas na forma de exercícios seriados para o aprendiz.

Assim sendo, o papel do controlador não deve ser apenas o de acesso por paginação fácil ao conteúdo mas também o de tornar disponíveis os aspectos metacognitivos envolvidos com as tarefas típicas de aprendizagem. Alguns autores [1] caracterizam os aspectos meta-cognitivos como um conjunto de elementos da interface por meio dos quais é possível ao aprendiz realizar atividades reflexivas que demonstrem algum grau de consciência e controle sobre o seu processo de solução de problema. Exemplos de tais atividades são as de marcar, sublinhar, colecionar e a de reverter alguns passos já realizados em direção à referida solução para que passos corretivos sejam adotados. Todavia, com a invenção de técnicas cada vez mais modernas de interatividade dos $\mathrm{OA}$, crescem também os desafios de criação e generalização das tarefas de natureza meta-cognitiva correspondentes. Tais desafios se manifestam de maneira particularmente acentuada quando o ambiente de execução inclui dispositivos manuais e de acesso móvel que permitem a aprendizagem em qualquer lugar, a qualquer hora [2].

Assim sendo, no processo de aquisição de conhecimento, seja ele mediado ou não pelo computador, é inevitável que o aprendiz cometa alguns erros. Os erros dos aprendizes são comuns, principalmente, durante a resolução de exercícios, como por exemplo, na resolução de uma expressão aritmética ou algébrica. É a partir desse ponto que pode ser motivada a reflexão do aprendiz sobre como ele abordou o problema proposto e se ele realmente possui o conhecimento necessário para resolvê-lo. Dessa forma, a partir dos erros é possível que o aprendiz amplie ainda mais seu conhecimento. Exemplos disso podem ser encontrados nos ambientes de aprendizagem baseados na arquitetura ACT* [3]. Nesses ambientes, um erro cometido leva o aprendiz a refletir e entender melhor suas ações e conceituações. A partir de uma abordagem sistemática, o ambiente consegue identificar se algo está incorreto no raciocínio do aprendiz durante a resolução de um problema. Caso algo inexato seja identificado, uma intervenção (feedback) imediata é mostrada ao aprendiz para que ele então possa tentar novamente e, eventualmente, encontrar soluções corretas a partir dos erros vistos.

Com isso, um exemplo de evento meta-cognitivo promovido pelo potencial de um módulo controlador de interatividade é a possibilidade de o aprendiz retroceder diretamente ao contexto de erros cometidos no passado, uma vez que ele perceba em um dado instante a razão genérica por trás de sua incompreensão. De acordo com estudos recentes [4], isso ajuda a promover condições para a reflexão sobre os passos adotados na solução de problemas e faz com que os mesmos caminhos de solução, ou novos caminhos, sejam tentados.

O desenvolvimento de software educacionais, geralmente é fundamentado em teorias que investigam como as pessoas aprendem e adquirem competências, além de investigar também como e porque elas cometem erros. Dentre essas teorias, as que mais se destacam são a ACT (Adaptive Control of Thought) [4], REPAIR [5], e STEP [6]. Essas teorias foram tradicionalmente utilizados para lidar com o erro em software de interface tutorial ativa, as quais são comumente caracterizadas como Sistemas Tutores Inteligentes (STI). Isso difere fundamentalmente da iniciativa proposta no presente trabalho, onde a abordagem é de interface passiva (i.e., dos Ambientes Exploratórios de Aprendizagem, ou AEA). Sendo assim, os recursos que apoiam a retroação direta do aprendiz a contextos de erros é influenciada por parâmetros ou propriedades do próprio erro. Uma das vantagens de tal representação está no fato de se codificar a ordem com que o acesso ao contexto passado do erro é oferecido ao próprio aprendiz que o cometeu, o que pode ser feito por meio de hipertextos (links) convencionais como os de qualquer máquina de busca da Web.

Porém, desenvolver software com esses potenciais não é um desafio simples, ainda mais quando eles são voltados à educação. Esse tipo de sistemas exige muita integração entre profissionais de diversas especialidades. Um exemplo desses profissionais são os programadores especializados, responsáveis pela implementação propriamente dita. Outros são os autores de conteúdo, que têm a tarefa de decidir a forma como esse conteúdo será apresentado assim como os problemas que o complementarão e fixarão tal conteúdo.

Dificilmente um programador possui amplo domínio do conteúdo que será apresentado pelo software educacional. De maneira semelhante, também não se deve esperar que o especialista do conteúdo saiba programar. Todavia, o programador, com seu conhecimento e experiência, pode propor um arcabouço genérico no qual os autores podem desenvolver seu conteúdo com pouco ou nenhum conhecimento de programação. Para tratar desse problema, surgiu a área de pesquisa de linguagens e ferramentas de autoria [7].

Com base na busca bibliográfica realizada, verificouse que ainda existe uma carência de abordagens 
conceituais e ferramentas de software destinadas a este propósito específico. Neste sentido, o contexto do erro se torna uma forma rica de aprendizagem exploratória livre (i.e., dos MEA), sem a intervenção frequente das máquinas de tutoria tais como os STI.

\section{Resenha Literária}

As linguagens de autoria podem ser classificadas como linguagens de programação peculiares. Elas são projetadas com o objetivo de proporcionar formas claras e de rápida assimilação para a construção de software educacional. Essas formas claras, na verdade, são Múltiplas Representações Externas (MRE) [8], conhecidas por elevarem o grau de abstração e de cobertura da descrição de conceitos de um domínio específico.

Criadas a partir de uma linguagem rica em MRE, as ferramentas de autoria são a implementação de compiladores ou interpretadores acoplados a ambientes de editoração. Em geral, tais ferramentas são utilizadas por meio de técnicas de programação visual no estilo WYSIWYG (What You See Is What You Get) de tal maneira que o autor não precise usar nenhum tipo de programação por de linhas de comandos.

Apesar de essa abordagem ser mais interessante do que escrever o código linha por linha, ela limita o usuário ao projeto do software [9], pois todas as decisões de como desenvolver um OA já foram tomadas por quem projetou a ferramenta. Como exemplos de sistemas de autoria voltados à criação de STI (Sistemas Tutores Inteligentes), pode-se citar os ambientes EON [7], RUI [10] e SIMQUEST [11].

Alguns ambientes de autoria do passado, como o RUI, tiveram o objetivo de ensinar apenas conceitos de subdomínios visuais especializados (e.g., Radiologia médica). Devido a isso, eles implementam apenas os modelos de domínio e pedagógico da arquitetura funcionalista interna de um STI. Outros foram mais genéricos, tal como o EON, e implementaram todos os quatro modelos especialistas da referida arquitetura de STIs, incluindo os modelos do aprendiz e da interação. O SIMQUEST, por sua vez, foi destinado à criação de simuladores baseados em ambientes exploratórios de aprendizagem.

Apesar das facilidades que esses sistemas de autoria oferecem, eles apresentam extrema rigidez gramatical em certas situações de editoração. No ambiente RUI, por exemplo, há a necessidade do autor conhecer sentenças em Lógica de Predicados, o que significa uma dificuldade para leigos. Adicionalmente, pouco foi relatado sobre o funcionamento do mecanismo essencial da shell interpretadora do modelo de aprendiz do ambiente EON. Quanto ao SIMQUEST, nenhuma pesquisa evidenciou o potencial dos seus aspectos metacognitivos que afetam o controle que o usuário final (aprendiz) tem sobre o acesso aos conteúdos inseridos pelo autor das simulações.

O desenvolvimento de qualquer software é uma tarefa complexa e em se tratando de software educacional, ela se torna ainda mais difícil por ser multidisciplinar. Geralmente, essa dificuldade é acentuada na hora da criação dos aspectos interativos controlados pelo aprendiz. Se eles não forem bem projetados do ponto de vista pedagógico, podem fazer com que o software tenha pouco valor educacional [12]. Tudo isso acaba caracterizando problemas de baixa eficiência [13] do ponto de vista de apoio à aprendizagem. Para que tais situações não aconteçam, deve existir um equilíbrio entre o potencial educacional e a atratividade do software.

Em resumo, quando se constrói um software educacional (incluindo a interface), ele deve ser apoiado em alguma suposição de como as pessoas aprendem. Por isso, deve-se tentar desenvolver software como um recurso para promover o crescimento pessoal, não deixando-o apenas com a visão do projetista mas principalmente com a do aprendiz. Assim sendo, a construção de interfaces voltadas à educação deve ter a preocupação de considerar, mesmo que simplificadamente, um perfil de longo prazo do aprendiz [14], fato que ainda é muito raro nos dias atuais mas que deve mudar muito nos próximos anos.

As limitações citadas no parágrafo anterior afetam até mesmo os mais famosos STI da história, que são ambientes inteligentes, conhecidos por realizarem o diagnóstico ativo de erros do aprendiz. Mesmo assim, nem os STIs possuem com frequência qualquer registro de desempenho para monitoramento de longo prazo do aprendiz em abordagens variadas de solução de problemas. De fato, pode-se dizer que os STIs desenvolvidos com base nas teorias ACT, REPAIR e STEP atuam de uma forma em que são capazes de fornecer apenas ajuda ao aprendiz, em formato textual, a curto prazo.

\subsection{Limitações do OAs para Ciências Físicas e Matemáticas.}

Atualmente, muitos dos OAs atuam de maneira puramente somativa quando conduzem algum processo de avaliação [15]. Em outras palavras, a finalidade desses OAs é estabelecer a porção de conhecimento adquirida por um aprendiz em um determinando domínio de especialidade.

Por outro lado, entre os OAs encontrados nesta pesquisa, são raros os que atuam de um maneira 
formativa [16, 17]. Ou seja, os que possuem a capacidade de constatar se realmente os aprendizes estão alcançando os objetivos pretendidos durante o processo de aprendizagem. Os OA com essa característica realizam avaliações contínuas sem se preocuparem com a atribuição de notas ou de determinar algum grau de aprendizado. Eles se preocupam em identificar as principais dificuldades dos aprendizes e tentam auxiliá-lo durante sua aprendizagem.

De forma resumida, OAs com caráter de avaliação somativa buscam auxiliar o julgamento de classificação dos aprendizes no final de uma determinada aprendizagem. Já os OAs com caráter formativo, acompanham todo o processo de aprendizagem fornecendo feedback sempre que acharem necessário. Com isso, os de caráter formativo induzem a reflexão do aprendiz sobre seu aprendizado e conduzem-no a um melhor desempenho [16]. Dessa forma os OAs formativos, apesar de raros, são os que possuem maior potencial de utilidade para as áreas de ciências físicas e matemáticas.

\subsection{Ambientes Exploratórios para Aprendizagem (AEA)}

No que diz respeito aos AEAs, pode-se defini-los como ambientes de auto-estudo, onde a aquisição de conhecimento se dá por atividades de exploração, investigação e descoberta. Apesar de não interagirem pró-ativamente com o aprendiz, esses ambientes mostram-se semanticamente ricos, pois oferecem diversos recursos que geralmente estão representados em formatos variados com o objetivo de aumentar a chance de assimilação do aprendiz por meio de metáforas computacionais bem construídas.

Um exemplo clássico de um ambiente exploratório é o ambiente LOGO [18]. Ele enfatiza que o erro do aprendiz não é algo negativo, mas sim uma forma de aprendizagem onde o conhecimento é construído com base em experiências no passado. Uma das maiores dificuldades no desenvolvimento de ambientes deste tipo está no momento da criação dos aspectos interativos controlados pelo aprendiz. Se eles não forem bem projetados do ponto de vista pedagógico, podem fazer com que o software tenha pouco valor educacional. Tudo isso acaba caracterizando problemas de baixa eficiência [25] do ponto de vista de apoio aprendizagem.

Uma das fragilidades encontras nesses ambientes está no fato que não possuem nenhum tipo de mecanismos de recuperação de erros. Alguns deles chegam a identificar os erros dos aprendizes mas, nem como interface passiva, são capazes de apresentar mensagens mais elaboradas aos aprendizes. Se isso fosse feito com certa frequência por tais ambientes exploratórios, daria um maior potencial de reflexibilidade ao aprendiz [19, 20, 21].

\section{Conceitos fundamentais adotados}

A abstração é o processo por meio do qual os aprendizes criam entidades mentais que servem como base para novos procedimentos e a formação de conceitos [8]. Nesse sentido, os aprendizes podem construir referências através das MREs para que a estrutura subjacente do domínio representado seja eventualmente exposta e tratada como um novo conhecimento, ainda mais genérico. Estudos passados demostram que a aprendizagem realizada com pares de representações externas leva a uma aquisição de conhecimento mais abstrato [22].

Neste projeto de pesquisa e desenvolvimento, a construção de conhecimento aprofundado foi planejada para ocorrer por meio de abstração por generalização. A partir disso, as MREs selecionadas nos diversos software do projeto são pelo menos em pares. Por exemplo, para o software de progressões geométricas (PG), elas são: (a) expressões analíticas organizadas passo-a-passo; (b) figuras da geometria fractal, as quais guardam correspondência com as várias versões de expressões do item anterior. É importante ressaltar que figuras de fractais não pertencem à geometria euclidiana clássica.

As figuras de fractais possuem estágios de formação que podem ser chamados de passos. Em cada um desses passos, as alterações geométricas provocam crescimento ou decréscimo em medidas de tamanho do menor lado, perímetro, sub-áreas circunscritas e alguns outros aspectos com potencial para refletir regularidades matemáticas tais como as propriedades de PG. Além disso, devido aos fractais não possuírem um número finitos de passos, seu uso pode auxiliar na aquisição de conceitos mais abstratos sobre infinitude e convergência em séries numéricas.

Nessa sequência de ideias, o aspecto mais importante para o desenvolvimento da capacidade de abstração está na maneira com que um aprendiz é levado a generalizar cada vez mais as expressões analíticas para que elas reflitam as grandezas de cada passo do fractal. Dessa forma, argumenta-se que um software pode levar à boa prática do desenvolvimento da capacidade de abstração do aprendiz se este último for envolvido com o reconhecimento e a descrição analítica precisa de representações passo-a-passo, tais como as da formação de fractais.

Mais especificamente, o presente projeto investigou como a generalização por indução poderia ser atingida tomando-se os passos de formação de figuras fractais 
como sendo compostas de progressões geométricas. Por definição, uma PG é uma sequência numérica em que cada termo, após o segundo, é igual ao produto do termo anterior por uma constante $q$. Por exemplo, a sequência $(1,2,4,8,16,32, \ldots)$ possui razão igual a 2 . Com essa definição em mente, pode-se observar a Tabela 1, a qual apresenta quatro passos da formação do fractal Triângulo de Sierpinsky. Tanto a quantidade de triângulos formados nos diferentes passos como o tamanho do menor lado de cada triângulo formam PGs diferentes. Na verdade, mais elementos matemáticos desse fractal formam PGs ao longo das transformações entre os passos. O objetivo da Tabela 1 é mostrar parte do processo de indução do aprendiz por meio de uma cobertura passo-a-passo que pode ser precisamente guiada com a ajuda de um software.

Na referida tabela, é possível observar a formação de ao menos duas PGs: uma crescente e outra decrescente. A primeira está relacionada com a quantidade de triângulos e a segunda com o tamanho do lado do menor triângulo, ambas consideradas em cada passo. Observe que, se o tamanho do lado do triângulo inicial for definido com o valor genérico $L$, a divisão sucessiva dos lados em seus pontos médios leva gradualmente à sequência:

$$
\frac{L}{2}, \frac{L}{4}, \frac{L}{8}, \frac{L}{16}, \ldots
$$

Esse processo pode ser repetido infinitamente, sendo que em cada passo, uma figura com triângulos cada vez menores será formada. Além disso, o importante mesmo do ponto de vista de generalização é que o aprendiz deverá descrever por si só o tamanho do lado no n-ésimo passo (passo de ordem $n$ ) como sendo:

\begin{tabular}{|c|c|c|c|}
\hline PASS & & NÚMERO DE TRIÂNGULOS & TAMANHO DO LADO DO TRIÂNGULO (16) \\
\hline $\boldsymbol{\Delta}$ & 1 & b & 8 \\
\hline An & 2 & 9 & 4 \\
\hline$\widehat{A}$ & 3 & 27 & 2 \\
\hline 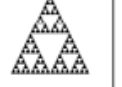 & 4 & 81 & 1 \\
\hline
\end{tabular}

Tabela 1: Passos de formatação do Triângulo de Sierpinsky

Essa representação passo-a-passo exige do aprendiz um desenvolvimento analítico de sua capacidade de abstração. Os conhecimentos matemáticos intrínsecos de um fractal fazem com que o aprendiz construa uma referência sobre os conceitos visualmente estruturados por bases analíticas. Isso parece tornar intuitivo o entendimento do assunto através do uso de MREs adequadas para serem manipuladas tanto por um software como por um humano, mantendo ainda a ligação direta com elementos concretos da rotina didática de solução de problemas (i.e., de Pgs e suas propriedades).

\section{Arquitetura Funcionalista}

Para consolidar os fundamentos de aprendizagem adotados até o momento no presente projeto de pesquisa e desenvolvimento, diversas ferramentas de software educacional foram implementadas e estão sendo ampliadas e validadas em ambientes escolares. A abordagem arquitetural de todas elas é baseada na existência de um núcleo comum de software, o qual é um arcabouço chamado aqui de Controlador de Acesso Reflexivo e Retroativo Indexado por Erros (CARRIE).

\subsection{Organização funcionalista do CARRIE}

Conforme representado na Figura 1, quatro módulos principais compõem a arquitetura funcionalista do CARRIE. São eles: Módulo de acesso ao conteúdo, Módulo indexador de erros, Módulo Facilitador e a Interface com o aprendiz. Todos esses módulos serão detalhados na sequência.

Foram implementados, completamente, quatro OAs que fazem uso do arcabouço CARRIE: (1) Progressões Geométricas em Fractais; (2) Funções de Primeiro Grau; (3) Matemática Financeira; (4) Funções Periódicas. Os 
quatro software educacionais fazem parte da fase inicial de construção e aplicação de apoio computacional a atividades laboratoriais do ensino escolar de conceitos matemáticos do nível médio. A iniciativa destinou-se ao cumprimento de metas no âmbito do Projeto CONDIGITAL [26], financiado com recursos do Edital conjunto MCT/MEC 001-2007.

Para a implementação do CARRIE, foi utilizada a plataforma Java seguindo as técnicas do paradigma de programação Orientada a Objetos. O software foi projetado para ser executado em qualquer navegador $W e b$, independente de sistema operacional ou hardware. Além disso, o código é divulgado como código livre sobre licença GPL (Licença Pública Geral).

\subsection{Módulo Indexador de Erros}

Este módulo é composto por 2 partes principais, o monitorador de erros e o guia de retroação.

\subsubsection{Monitorador de Erros}

O monitorador de erros funciona com base em pontos de observação definidos pelo autor do conteúdo. Mais precisamente, o autor precisa necessariamente marcar, através da chamada de um método, onde o aprendiz comete um erro. Os parâmetros desse método são o título do erro, o texto explicativo do erro e uma variável buleana. Quando essa variável for verdadeira, uma janela popup é mostrada, alertando o aprendiz para o erro. A área visual do popup na tela tem como conteúdo o título e o texto explicativo, passado como parâmetro para o método. Caso seja falsa, nada será mostrado, apenas o estado da aplicação será salvo.

No momento que esse método é chamado, o monitorador de erros recebe uma mensagem indicando um desvio conceitual cometido pelo aprendiz. Após o recebimento dessa mensagem, o monitorador salva o erro e o estado da aplicação em que o erro ocorreu em um banco de dados interno. Com essas informações salvas, o CARRIE estrutura o seu comportamento para que o aprendiz possa retroceder ao momento exato em que o erro foi cometido. Sendo assim, é apresentado ao aprendiz um guia de retroação, por meio do qual o aprendiz pode retroceder a qualquer erro cometido durante seu período de aprendizado.

\subsubsection{Guia de Retroação}

O guia de retroação permite que o aprendiz inspecione momentos exatos de erros passados e decida a quais deles vale a pena retroceder. Este guia faz a leitura do banco de dados interno de erros e cria um menu contendo um link para cada erro armazenado no banco de dados. Esse menu é mostrado ao aprendiz somente depois de existir pelo menos um erro cometido. Quando o menu está visível, o aprendiz pode visualizar o título do erro e o seu texto explicativo. Passando-se o mouse sobre o link do erro, a qualquer momento pode-se retroceder ao erro cometido apenas clicando no link.

Quando o aprendiz retrocede ao erro, o CARRIE possibilita que ele refaça todo o exercício em que o erro foi cometido. Tal abertura de tarefas promove fundamentalmente o que é chamado de atividade reflexiva e permite que o aprendiz tente revisitar os aspectos que o levaram ao erro. Caso o aprendiz não queira refazer todo o exercício novamente (e.g., por sentir que ainda tem dúvidas), ele também pode desistir da tentativa. Vale a pena ressaltar aqui que, ao contrário dos tradicionais recursos de desfazer simplesmente uma tarefa (undo, em inglês), o guia de retroação atinge um potencial meta-cognitivo muito mais adequado à finalidade pedagógica do CARRIE. Acredita-se que essa característica lhe confere um bom grau de originalidade em relação aos ambientes existentes.

\subsection{Módulo de Acesso ao Conteúdo}

É um conjunto de módulos responsáveis por apresentar o conteúdo ao aprendiz. Ele está dividido nos seguintes sub-módulos.

\subsubsection{Glossário de Termos}

O CARRIE oferece um módulo para a criação do glossário de termos do domínio do OA de uma maneira simples e clara. Quando o autor achar necessário que um glossário de termos seja disponibilizado ao aprendiz, ele somente precisa definir um arquivo respeitando uma indentação pré-estabelecida. Quando o CARRIE identificar que um arquivo que segue essa estrutura foi criado, ele automaticamente disponibilizará a interface responsável pela apresentação do glossário por meio do link de acesso. Além disso, o glossário ainda conta com uma busca por palavra-chave baseada no recurso de auto-completar. 


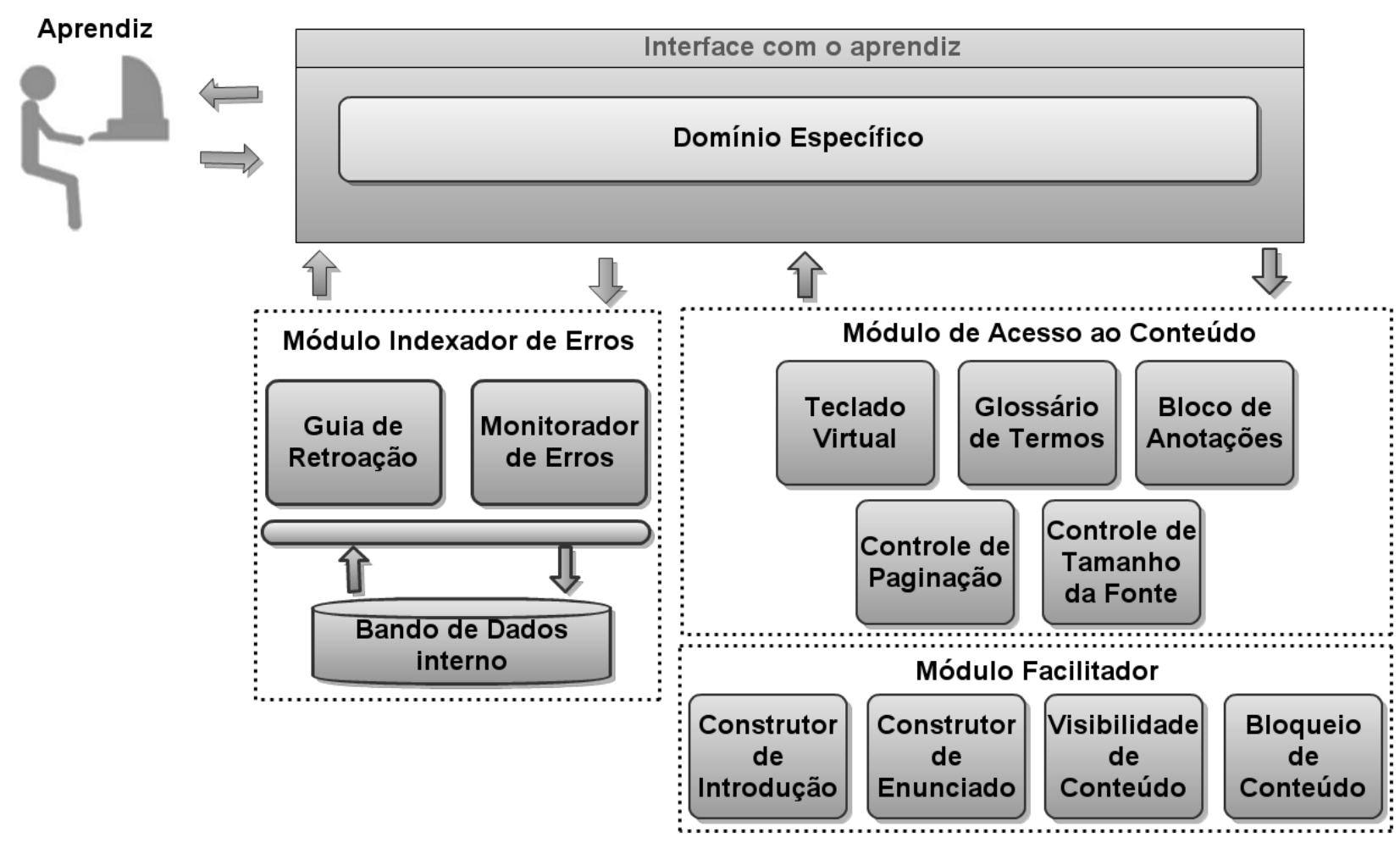

Figure 1: Arquitetura funcionalista do CARRIE

\subsubsection{Controle de Tamanho de Fonte}

Um aspecto importante para os software atuais é a acessibilidade. Devido a isso, o CARRIE oferece um controle para que o aprendiz atue sobre o tamanho de letras de qualquer texto do OA.

\subsubsection{Controle da Paginação}

O CARRIE tem como um dos objetivos fazer com que o aprendiz desenvolva seu conhecimento passo-apasso para atingir abstração por generalização. Para que isso ocorra, há um módulo que é responsável por oferecer paginação de todo e qualquer conteúdo a ser apresentado. Em outras palavras, o autor de material eletrônico não precisa se preocupar com esse tipo de código, pois ele está disponível no arcabouço.

\subsubsection{Teclado Virtual}

Alguns OAs necessitam manipular uma entrada de dados do tipo numérico-analítica. Para isso, é necessário um estilo diferente de mecanismo de interação. Esse mecanismo é um teclado virtual, que é disponibilizado pelo CARRIE para o autor do conteúdo fazer uso em seu OA. Ele está ilustrado na Figura 2. O teclado virtual usa a metáfora de uma calculadora científica estendida, onde é possível representar expressões mais complexas com a visualização bidimensional imediata da mesma. No caso de todos os OA deste padrão, é possível a entrada de expressões em função das variáveis $l$ e $n$, usando, além das quatro operações normais e parênteses, potenciação e radiciação.

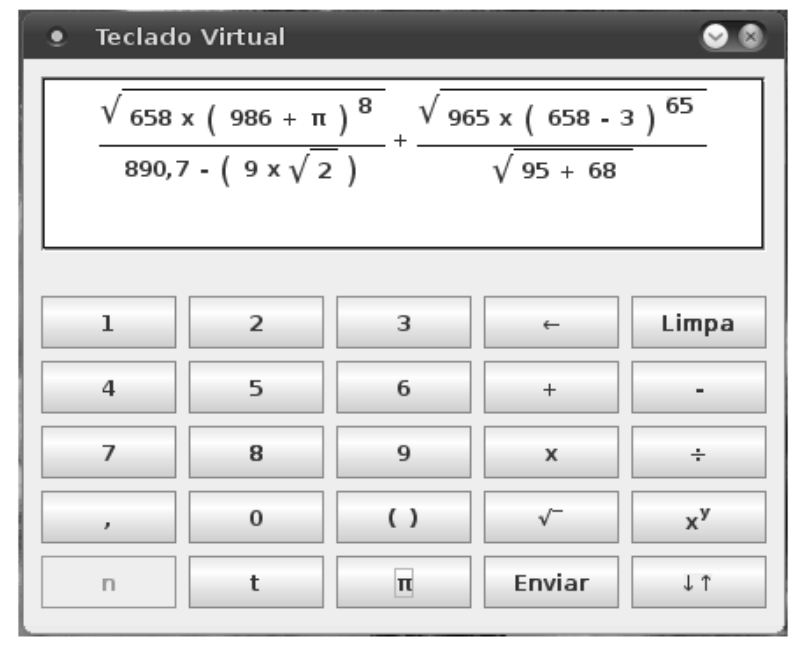

Figura 2: Teclado Virtual para a entrada de dados numérico-analítticos

\subsubsection{Bloco de Anotações}

Um aspecto que pode ser considerado importante 
para o aprendizado é o bloco de anotações. Nele o aprendiz pode realizar anotações de todo o conteúdo que achar importante e, dessa forma, pode construir sua própria percepção do tema em foco. Além disso, um bloco de anotações pode facilitar a organização dos conteúdos que merecem mais atenção por parte do aprendiz. A partir disso, o CARRIE oferece um bloco de anotações ao aprendiz com recursos para destacar o texto em diversas cores.

\subsubsection{Calculadora}

Outro recurso importante para os software educacionais voltados às áreas de ciências físicas e matemáticas é a calculadora. Com isso, o CARRIE oferece uma calculadora para o aprendiz. Ela pode ser usada de maneira clara e simples.

\subsection{Módulo de Acesso ao Conteúdo}

Os sub-módulos contidos no módulo facilitador são destinados a tornar o desenvolvimento de um OA, com o uso do CARRIE, mais simples e com menos preocupação em codificação. Para isso, o CARRIE oferece três submódulos:

- Construtor de Introdução;

- Construtor de Enunciado;

- Bloqueio de conteúdo e Visibilidade de Conteúdo.

\subsubsection{Construtor de Enunciado}

Da mesma maneira que o autor do conteúdo pode formatar seu texto para introdução, ele também pode formatar seu texto para o enunciado. Através do CARRIE, é possível construir um enunciado seguindo o padrão de formatação e apresentação de texto em HTML. Além disso, também é possível ligar palavras do enunciado com sua definição no glossário de termos. Além disso, este módulo oferece uma opção na qual o aprendiz pode esconder e mostrar o texto do enunciado a qualquer momento. Dessa forma, pode-se aproveitar melhor o espaço disponível na tela do computador para resolver o exercício.

\subsubsection{Bloqueio e Visibilidade de um Conteúdo}

Certos conteúdos podem ser bloqueados ou escondidos pelo autor para que o aprendiz os acesse somente quando for apropriado. Isso oferece controle indireto do professor sobre os alunos espalhados no laboratório por meio de um módulo que garante que um exercício mais fácil é resolvido antes de outro substancialmente mais difícil.

\subsection{Interface com o Aprendiz}

Uma interface com o aprendiz é pré-estabelecida pelo CARRIE e está dividida em três partes principais. A primeira é destinada ao título do OA, juntamente com o título da página atual. Em seu canto superior direito, também estão as opções para aumentar e diminuir o tamanho das letras. A segunda parte é destinada ao domínio específico. A outra é reservada às opções da dinâmica de controle oferecida ao aprendiz. Exemplos de tais controles são: paginar para frente ou para trás, botão de acesso ao glossário, botão de acesso ao guia de retroação e o botão de acesso ao bloco de anotações.

A Figura 3 mostra aspectos gráficos da interface em um certo instante da interação com o OA de Funções Lineares sobre força e deslocamento de molas. No quadro estão ressaltados como as constantes elásticas das molas influenciam os gráficos das funções de primeiro grau de cada uma delas.

\section{Perspectivas sobre o mecanismo de retroação a erros}

Atualmente o CARRIE tem o objetivo de oferecer um arcabouço para o desenvolvimento de OAs destinados à utilização em laboratórios durante o período de uma ou duas aulas de 50 minutos. Devido a isso, o registro do estado exato do erro pode ser feito apenas na memória de curto prazo. Ou seja, o aprendiz e o professor terão acesso aos erros cometidos apenas durante a execução corrente do OA. Em outras palavras, o sistema não permite que esses erros sejam acessados e analisados em outra sessão de execução do OA.

Consequentemente, tanto $\mathrm{o}$ aprendiz quanto $\mathrm{o}$ professor ficam limitados a um período pequeno de tempo para a análise e discussão dos erros. O que de fato seria mais interessante e produtivo do ponto de vista pedagógico é oferecer acesso aos erros a qualquer momento além do tempo de execução, como por exemplo, dias ou meses depois deles terem ocorrido. Com isso, o professor poderia reavaliar o aprendizado dos alunos, acessando seus erros, e identificar em quais partes do conteúdo apresentado o aprendiz teve mais dificuldade. Além disso, de posse dessas informações, o professor também poderia oferecer mecanismos mais direcionados para recuperar as falhas do aprendiz para que este consiga ampliar seu conhecimento de maneira mais sistemática.

Nesse mesmo sentido, o aprendiz também não ficaria limitado a rever seus erros apenas no período da execução do OA. Isso permitiria um aumento do potencial de auto-identificação de falhas conceituais por 


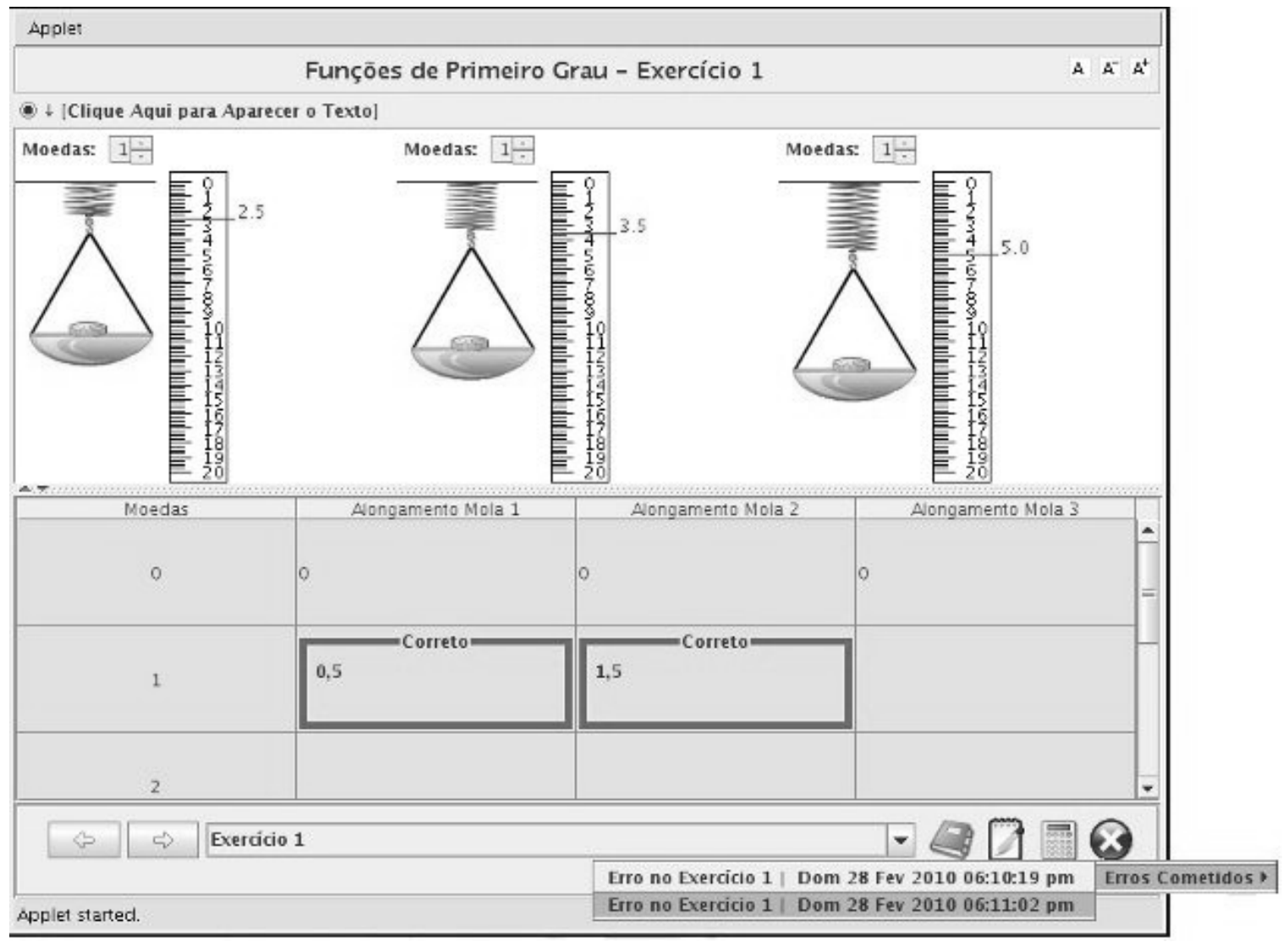

Figura 3: Interface do OA sobre funções de primeiro grau.

parte do aprendiz, ou ainda com a ajuda do professor. Com isso, o aprendiz poderia se aprofundar mais do que apenas identificar as causas de seus erros. Ele teria a possibilidade de refazer, a qualquer momento, todo o exercício no qual o erro ocorreu, certificando-se assim que realmente compreendeu o conteúdo apresentado.

A partir disso, uma nova versão do CARRIE está sendo desenvolvida com o objetivo de salvar os erros dos aprendizes a longo prazo. Sendo assim, além dos benefícios supracitados, de posse de uma base de dados de erros, seria possível realizar análises automáticas sobre os erros cometidos e determinar as principais dificuldades encontradas por cada aprendiz, individualmente, ou por um grupo de aprendizes. Com isso, quando o aprendiz for rever um erro. o software pode realizar a análises de todos os dados e gerar informações mais precisas sobre os erros dos aprendizes.

Mas para que isso seja possível, é necessário expandir a representação de erros interna e também a externa. E consequentemente seria necessário construir uma representação taxonômica do erros com o objetivo de classificá-los de acordo com algumas categorias estabelecidas na taxonomia. A partir disso, seria possível expandir o mecanismo de busca por erros cometidos, para este seja mais eficiente e traga resultados relevantes de acordo com os parâmetros requisitados pelos aprendizes e professores.

Com a construção desse módulo, em princípio também seria possível modelar e desenvolver um interpretador do modelo do aprendiz a longo prazo. De acordo com os dados capturados pelo software durante diversas interações com o aprendiz, ele poderia estimar com certa precisão quais são as dificuldades dos aprendizes e que assuntos eles devem estudar antes de prosseguirem a solução de novos problemas.

\section{Considerações Finais e Trabalhos Futuros}

Neste trabalho foram apresentadas tanto a necessidade como a proposta de um controlador de interatividade genérico que evidencia os aspectos metacognitivos envolvidos em tarefas típicas de aprendizagem. Além disso, foi evidenciada uma das principais limitações dos OA, que é a ausência de uma forma de utilizar o erro do aprendiz como uma maneira eficiente de mecanismo reparador e de aquisição de conhecimento. Ressaltou-se também, neste documento, a 
ausência de pesquisas no que diz respeito a este nicho específico de tratamento de contextos de erro por parte do próprio aprendiz humano.

Destacou-se na resenha literária os aspectos concernentes a Ambientes Exploratórios de Aprendizagem assim como às diretrizes fundamentais para o desenvolvimento de interfaces educacionais. Coube também a essa parte elucidar os aspectos que dizem respeito às linguagens e ferramentas de autoria. Finalmente, ainda na resenha, enfocou-se as principais limitações dos objetos de aprendizagem para ciências físicas e matemáticas.

Para demostrar o conceito de conhecimento aprofundado através da abstração por generalização, foi descrito o uso de fractais para o ensino de progressões geométricas e sua extensão para outros domínios por meio de uma arquitetura genérica.

Para consolidar os fundamentos de aprendizagem adotados no presente projeto de pesquisa $\mathrm{e}$ desenvolvimento, apresentou-se na Seção 4 uma abordagem arquitetural caracterizada por um núcleo comum para o desenvolvimento de diversos software educacionais. $\mathrm{O}$ arcabouço é chamado de Controlador de Acesso Reflexivo e Retroativo Indexado por Erros (CARRIE).

De acordo com resultados obtidos por este projeto de pesquisa e desenvolvimento, as perspectivas futuras apontam naturalmente para um maior aprofundamento das formas de retroação às situações onde o aprendiz cometeu erros. A ideia de registrar e restaurar os quadros de erro ainda é feita de maneira quase linear no arcabouço atual do controlador CARRIE. Adicionalmente, a iniciativa mais próxima de pesquisas do passado que ofereceram ao aprendiz uma visão de ambientes de aprendizagem com recursos para os usuários inspecionarem o que o software assumiu sobre eles foi chamada de modelos abertos de aprendizes (open learner models) [23]. Nessa categoria de sistemas tutores, o conceito de skillometer [24] como marcador de valor em uma escala de habilidade foi uma das formas com que os modelos abertos de aprendizes mais se projetaram no mundo de pesquisa.

No entanto, tais iniciativas não contemplaram a criação e o uso de linguagens de descrição da configuração com que os erros do aprendiz ocorreram para que tais erros pudessem ser revisados em outras condições no futuro. Essa fronteira de pesquisa continua totalmente inexplorada e se constitui em um campo relevante de interesse teórico e prático. Seus conteúdos cobrem desde aspectos epistemológicos da representação do conhecimento humano sobre os estados do mundo, até detalhes de implementação que referenciam o chamado cálculo de situações no clássico mundo de blocos [25].
Por si só, a abordagem integradora de tais conteúdos sob uma linguagem única representa um grande desafio de pesquisa, cuja complexidade certamente contribuirá com conhecimentos originais para a Informática na Educação.

Além disso, como perspectivas futuras podemos citar expansão do mecanismo de retroação para não apenas dar a possibilidade do aprendiz voltar ao erro, mas também para o OA direcionar o aprendiz em quais assuntos, sejam mais específicos ou mais gerais, ele deve ter mais atenção para obter um compreensão clara dos conceitos abordados. No mesmo sentido, busca-se também validar e aperfeiçoar essa pesquisa por meio de uma aplicação da ferramenta em ambiente realista do ensino em escolas.

Agradecimentos. O projeto é financiado pela iniciativa de âmbito nacional intitulada CONDIGITAL, com recursos de FNDE (Fundo Nacional para Desenvolvimento da Educação) por meio do Edital 001/07 MEC/MCT. Os autores do presente artigo agradecem aos seus parceiros de consórcio (LACTECInstituto de Tecnologia para o Desenvolvimento, UELUniversidade Estadual de Londrina e DITEC/SEED/PRDiretoria de Tecnologias Educacionais da Secretaria de Estado da Educação do Paraná) por conduzirem outros projetos complementares em outras mídias que não são software.

\section{Referências}

[1] Puntambekar, S., Stylianou, A., and Hubscher, R. (2003). How do students navigate and learn from nonlinear science texts: Can meta navigation support promote learning? New Technologies And Their Applications in Education, 1(4):674-684.

[2] Sharples, M. and Beale, R. (2003). A technical review of mobile computational devices. J. Comp. Assisted Learning, 19(3):392-395.

[3] Anderson, J. R. (1983). The architecture of cognition / John R. Anderson. Harvard University Press, Cambridge, Mass.

[4] Bull, S. and Kay, J. (2007). Student models that invite the learner in: The SMILI open learner modelling framework. International Journal of Artificial Intelligence in Education, 17(2):89120.

[5] Brown, J. S. and VanLehn, K. (1988). Repair theory: A generative theory of bugs in procedural skills. In Collins, A. and Smith, E. E., editors, Readings in Cognitive Science: A Perspective from Psychology and Artificial Intelligence, pages 338-361. Kaufmann, San 
Mateo, CA.

[6] Vanlehn, K. (1987). Learning one subprocedure per lesson. Artif. Intell., 31(1):1-40.

[7] Murray, T. (1998). Authoring knowledge based tutors: tools for content, instructional strategy, student model, and interface design. Jrnl. of the Learning Sciences, 7:5-64.

[8] Ainsworth, S. (2006). Deft: A conceptual framework for considering learning with multiple representations. Learning and Instruction, 16(3):183-198.

[9] Batista, L., Direne, A., Trindade, J., Gimenes, I., and Taha, O. (2003). Autoria de diretrizes pedagógicas destinadas ao treinamento das múltipla capacidades da perícia em conceitos visuais. In SBIE2003 - Simpósio Brasileiro de Informática na Educação, pages 573-582.

[10] Direne, A. (1997). Designing intelligent systems for teaching visual concepts. Int. Jrnl of Artificial Intelligence in Education, (8):44-70.

[11] Van Joolingen, W. R., King, S., and Jong, T. (1997). The simquest authoring system for simulation-based discovery learning. In $\mathrm{du}$ Boulay, B. and Mizoguchi, R., editors, Frontiers in Artificial Intelligence and Applications: Proceedings of World Conference on Artificial Intelligence in Education (AI-ED 97), pages 7986. IOS Press.

[12] L. S. Fernandes, A. L. A. R. and Benitti, F. B. V. (2004). Interface de software educacional: Desafios de design gráfico. IV Congresso Brasileiro de Computação CBComp 2004, Informática na Educação, (3):254-258.

[13] Alves, G. (2007). Um estudo sobre o desenvolvimento da visualização geométrica com o uso do computador. In Simp. Bras. de Informática na Educação, pages 3-12.

[14] O'Shea, T. (1997). A typology for educational interfaces. In CHI '97: CHI '97 extended abstracts on Human factors in computing systems, pages 119-120. ACM.

[15] Márcia G. de Oliveira, Elias Oliveira, R. Z. M. (2009). Um qasystem para interação de alunos em avaliações somativas a distância. In XX SBIE - Simpósio Brasileiro de Informática na Educação (SBIE-2009), Florianópolis-SC, Brasil. SBC.

[16] Pimentel, E., Alves1, A. S., Oliveira, B. W. R., Ikebara, D. M., Bottaro, P. A., and Lopes, R. (2007). Avaliações adaptativas baseadas no nível de aquisição de conhecimentos do aprendiz. In XVIII SBIE - Simpósio Brasileiro de Informática na Educação (SBIE- 2007), São Paulo, Brasil. SBC.

[17] Zanella, R. (2005). Easy : sistema de avaliações via web baseado no hyper-autonomaton. Master's thesis, Universidade Federal do Rio Grande do Sul. Instituto de Informática.. Programa de Pós-Graduação em Computação.

[18] Papert, S. (1980). Mindstorms: Children, Computers, and Powerful Ideas. Basic Books, New York.

[19] Puntambekar, S. and Hübscher, R. (2005). Tools for scaffolding students in complex learning environments: What we have gained and what we have missed? Educational Psychologist, 40:1-12.

[20] Puntambekar, S. and Stylianou, A. (2003). Designing metacognitive support for learning from hypertext: What factors come into play? AIED

[21] Puntambekar, S., Stylianou, A., and Hübscher, R. (2003). Improving navigation and learning in hypertext environments with navigable concept maps. Hum.-Comput. Interact., 18(4):395-428.

[22] Schwartz, D. L. (1995). The emergence of abstract representations in dyad problem solving. The Journal of the Learning Sciences, 4(3):321-354.

[23] Diego Zapata-Rivera e Jim E. Greer. Exploring various guidance mechanisms to support interaction with inspectable learner models. Proceedings of the Conference on Intelligent Tutoring Systems (ITS-2002), pages 442-452, 2002.

[24] S. B. Blessing. A programming by demonstration authoring tool for model-tracing tutors. International Journal of Artificial Intelligence in Education, 8:233-261, 1997.

[25] G. Alves. Um estudo sobre o desenvolvimento da visualização geométrica com o uso do computador. SBIE2007 - Simpóio Brasileiro de Informática na Edução, páginas 3 -12, 2007.

[26] A. Direne, A. Pimentel, G. Ramos, D. Marczal, E. Carvalho, M. Castilho. L. García, F. Silva, L. Bona, M. Sunyé. Anais do XV Workshop sobre Informática na Escola (WIE-2009). In Anais do XV Workshop sobre Informática na Escola (WIE-2009), 1693-1702, Bento Gonçalves - RS. 